\title{
Judgments of relative time-to-contact of more than two approaching objects: Toward a method
}

\author{
PATRICIA R. DELUCIA and JENNIFER BLUME NOVAK \\ Texas Tech University, Lubbock, Texas
}

\begin{abstract}
Observers reported which of as many as eight computer-generated approaching objects would "hit" them first. Accuracy was above chance probability except when two-object displays contained pictorial relative size information that contradicted relative time-to-contact (TTC) information. Mean $d^{\prime}$ and response time was greater, but mean efficiency (Barlow, 1978) was smaller with eight objects than with two. Performance was less effective when global expansion contradicted TTC information than when local expansion contradicted TTC. Results suggest that observers can judge relative TTC with as many as eight objects when certain sources of information are consistent with TTC and that observers rely on information other than, or in conjunction with, optical TTC. Also, the sources of visual information that affect performance may vary with set size, and identification (but not detection) judgments may be constrained by limited-capacity processing.
\end{abstract}

Information in the optic array specifies when an object will hit or pass the observation point and when it will hit another designated object (Bootsma \& Oudejans, 1993; Lee, 1974; Tresilian, 1990, 1991). Results suggest that observers can use or are sensitive to such optical information about time-to-contact (TTC; e.g., tau, Lee, 1974)। in various tasks (see, e.g., Bootsma \& Oudejans, 1993; Kaiser \& Mowafy, 1993; Schiff \& Detwiler, 1979; Schiff \& Oldak, 1990; Todd, 1981).

For example, Schiff and colleagues (Schiff $\&$. Detwiler, 1979; Schiff \& Oldak, 1990) measured judgments about TTC with a prediction-motion (PM) task in which a filmed or animated object approached the observation point and then disappeared from view. The observers pressed a button when they thought the object would have reached them had the object continued approaching at the same speed after it disappeared. Estimates of TTC increased as actual TTC increased, suggesting that judgments were based on optical TTC. Moreover, in another study, when observers judged which of two computersimulated approaching objects would contact the observation point first, they achieved over $90 \%$ accuracy when there was only a 150 -msec difference in TTC between the objects (Todd, 1981; see also Simpson, 1988).

Parts of this study were reported at the 1994 annual meeting of the Association for Research in Vision and Ophthalmology. We thank James R. Tresilian for suggesting the analyses of efficiency and local and global expansion. We are grateful to Norma V. S. Graham for valuable discussions and suggestions regarding data analyses and signal detection analyses. We also thank Robert W. Bell, Lanie A. Dornier, Mary K. Kaiser, David J. Law, and an anonymous reviewer for helpful comments on earlier drafts, and Jeff Maresh and Robert Todd of Engineering Solutions, Inc., for technical assistance. This research was supported by the Research Enhancement Fund at Texas Tech University. Correspondence should be addressed to P. R. DeLucia, Texas Tech University, Department of Psychology, Lubbock, TX 79409-2051 (e-mail: djxpd@ttacs.ttu.edu).
However, results also suggest that judgments about TTC are constrained by scene and threshold factors and can be based on multiple sources of information (see, e.g., DeLucia, 1991a, 1991b, 1995; DeLucia \& Warren, 1994; Law et al., 1993; Schiff \& Detwiler, 1979; Schiff \& Oldak, 1990; Tresilian, 1995). For example, results of the previously mentioned PM task indicated that observers estimated TTC inaccurately when actual TTC exceeded $2 \mathrm{sec}$, and that the inaccuracy increased as actual TTC increased; the majority of judgments were underestimations (Schiff \& Detwiler, 1979; Schiff \& Oldak, 1990). Further, TTC judgments were more accurate with lateral motion than with approach motion (Schiff \& Oldak, 1990). Other studies indicated that motion trajectory can also affect the accuracy of relative TTC judgments-that is, judgments about which of two objects would hit a target first (Bootsma \& Oudejans, 1993; Law et al., 1993) or about whether two objects would collide with each other (DeLucia, 1995).

Moreover, the finding that observers' judgments about TTC are consistent with their use of optical TTC does not preclude the possibility that such judgments are constrained by cognitive operations, especially in complex situations when several objects are judged or when objects are not continuously in view. Tresilian (1995) argued that task variables affect whether performance involves cognitive information processing and whether optical TTC is used. He proposed that PM tasks, but not fast-interceptive actions, may involve cognitive operations. One of the features of PM tasks that implicates cognitive operations is that the response occurs after the object disappears, in the absence of visual information (Jagacinski, Johnson, \& Miller, 1983; Schiff \& Oldak, 1990; Tresilian, 1995). For example, observers may cognitively extrapolate the object's motion after it disappears (DeLucia \& Liddell, in press; Schiff \& Oldak, 1990; Tresilian, 1995).

Tresilian's (1995) analysis also suggests that the influence of cognitive factors may be minimized in relative 
TTC judgments, compared with TTC judgments measured in PM tasks, because relative TTC judgments do not require a timed response. Therefore, potential effects of the time between the object's disappearance and the observer's response, and thus cognitive extrapolation of motion, can be minimized. Indeed, it has been reported that twoobject relative TTC judgments may rely on separate abilities from those governing single-object absolute TTC judgments (Law et al., 1993) and that performance on the two tasks is not correlated (Hunt, Pellegrino, Frick, Farr, \& Alderton, 1988).

However, although relative TTC tasks may minimize the use of cognitive motion extrapolation, other cognitive operations may affect such judgments. For example, when computer-generated floating objects approached an observation point, observers reported that a large far object appeared to hit the observation point before a small near object that was specified to arrive sooner by TTC information (DeLucia, 1991a, 1991b). Judgments were consistent with pictorial depth information of relative size rather than with TTC information. Such size-arrival effects occurred with an active collision-avoidance task (DeLucia \& Warren, 1994) and with judgments about whether two moving objects would collide with each other (DeLucia, 1995), and can be interpreted as implicating cognitive operations (see Tresilian, 1995).

Moreover, in prior studies of relative TTC judgments, displays contained only two objects (e.g., Bootsma \& Oudejans, 1993; DeLucia, 1991a, 1991b; Law et al., 1993; Simpson, 1988; Todd, 1981). ${ }^{2}$ Judgments of such simple displays may not reflect limits in cognitive processing such as attention and memory. For example, due to limits in visual acuity, all objects in a scene cannot be seen clearly at once. Observers must scan scenes with successive fixations and may rely on memory to integrate information across the fixations (Hochberg, 1978; Palmer, 1990). Similarly, observers may divide their attention among multiple items in a scene. However, capacity limits in memory and attention (see, e.g., Cowan, 1988; Kahneman, 1973; Shiffrin, 1976) may constrain an observer's ability to process information about scenes and thus to judge TTC. Law et al. (1993) suggested that relative TTC judgments require resource-limited central processing and that visual scanning and processing demands play a role in such judgments.

Thus, the primary objective of the present study was to measure relative TTC judgments of more than two approaching objects. We also began to evaluate whether such judgments are consistent with limited-capacity cognitive processing. We focused on two questions: (1) Can observers judge which of as many as eight approaching objects would hit them first at levels that are better than chance probability? (2) Does response time (RT) increase (and/or effectiveness decrease) as the number of approaching objects, or set size, increases? An increase in RT as a function of set size would be consistent with limitedcapacity processing (see, e.g., Townsend, 1974). ${ }^{3}$ The theoretical implication is that processing load as well as optical expansion information must be included in models of perceived collision (DeLucia \& Blume, 1994).

The design of displays in which more than two objects approach the observation point is complex and requires numerous constraints and controls. Nevertheless, this initial attempt to measure relative TTC judgments of as many as eight approaching objects produced systematic and informative results indicating that further studies of this kind would be worthwhile.

\section{EXPERIMENT 1}

\section{Method}

Observers. The observers were 8 students at Texas Tech University who had normal or corrected visual acuity and who were naive as to the experimental hypotheses. Seven students received credit toward an introductory psychology course and 1 received $\$ 10$.

Displays. Computer simulations of approaching objects were motivated by prior studies of relative TTC judgments of two approaching objects (DeLucia, 1991a, 1991b; Simpson, 1988; Todd, 1981). Simulations were created with a MS-DOS 486/50-Mhz computer and were presented in $640 \times 350$ pixel resolution at a speed of 23.4 frames/sec on a 35.56-cm (14-in.) monitor. Displays consisted of perspective white-on-black drawings of 3-D scenes in which either two, four, six, or eight objects approached the viewer for $2.48 \mathrm{sec}$. Schematic representations are shown in Figure 1. The optical parameters of the objects are shown in Table 1.

The object with the smallest TTC - the first-arriving object (FA) had a TTC of .7 sec on the last frame of the scene; the remaining objects had TTCs of .9, 1.1, 1.3, 1.5, 1.7, 1.9, and $2.1 \mathrm{sec}$. Thus, the minimal difference in TTC between FA and the other objects was $200 \mathrm{msec}$, which is above previously reported thresholds for comparable two-object relative TTC judgments (Simpson, 1988; Todd, 1981). ${ }^{4}$ Regardless of set size, the TTC of the FA was . $7 \mathrm{sec}$ and the TTC of the next-arriving object (NA) was $9 \mathrm{sec}$. That is, their spacing remained the same. This was necessary to avoid confounds between set size and differential TTC; judgment accuracy of twoobject displays varies with the latter (see, e.g., Todd, 1981).

The scene was divided into eight areas with boundary lines, and a maximum of one object was located in each area throughout a trial. ${ }^{5}$ The display area in which the FA and the other objects were located varied randomly across trials. We achieved this by assigning the FA to each of the eight locations to create eight separate scenes. Then, for each of these scenes, we assigned the remaining objects randomly to the unfilled locations with the constraint that each object appeared in each location once. The scenes were presented in random order.

Thus, we created eight unique scenes that each contained eight objects. These scenes were only a subset of the numerous scenes that can be created with all possible arrangements of the eight objects and eight display locations (e.g., the location of the FA was not completely crossed with the location of the other objects). We note that when the display location of the FA varied, the locations of the remaining objects also varied. Thus, the independent variable that we refer to as "FA location" could also be referred to as "scene." We identify scenes by the FA's location because the observer's task was to report the location of the FA and because we manipulated specific aspects of the FA (discussed below).

Finally, to create scenes with two objects, we modified each of the eight-object scenes by deleting six objects that had TTC values that were greater than the TTC of the NA. That is, we retained only the FA and the NA. Other aspects of the displays remained constant (e.g., the objects' positions, velocities, differential TTC). In fourand six-object scenes, objects were deleted similarly.

It was necessary to constrain the scenes in several ways to accommodate more than two approaching objects while minimizing 

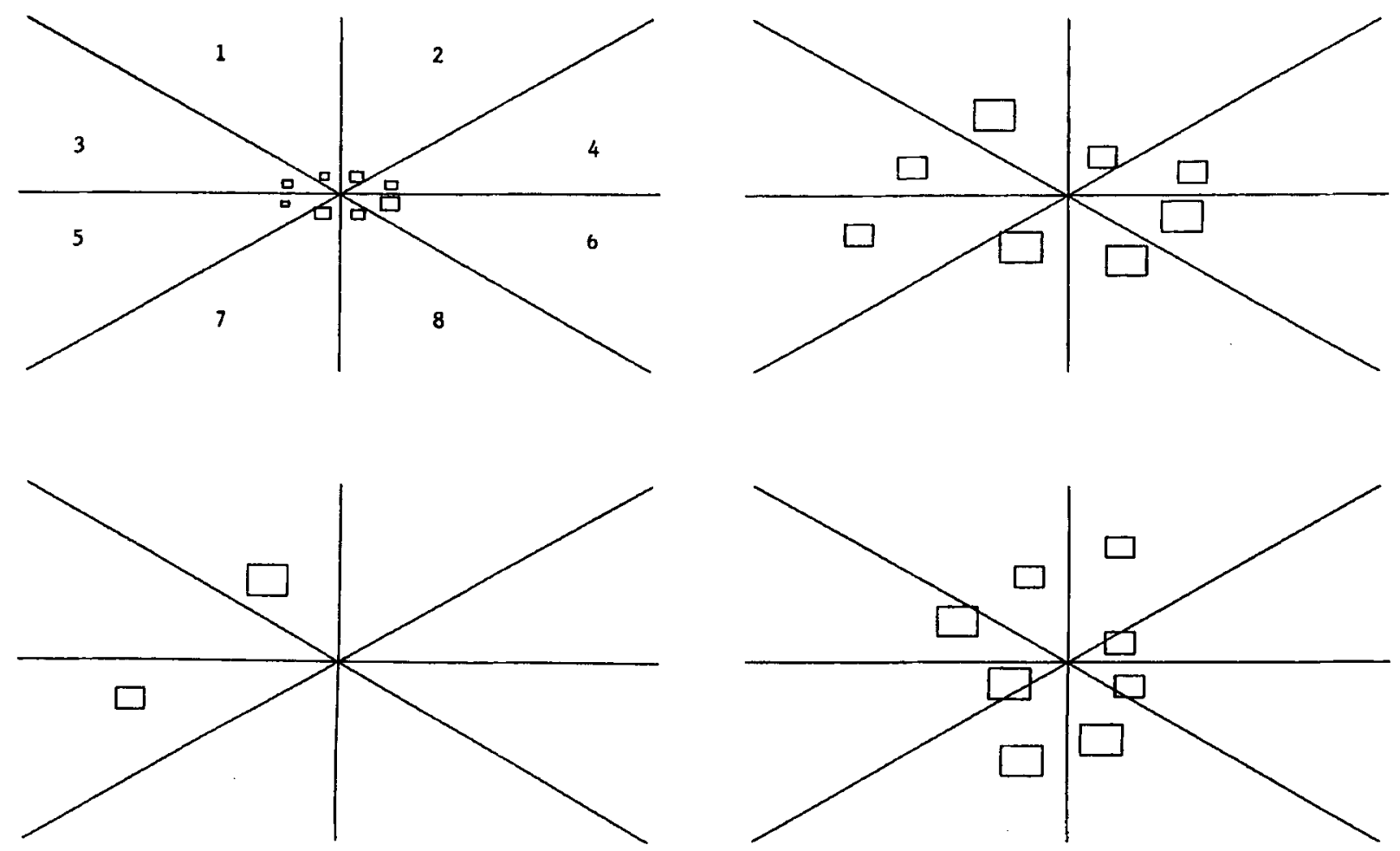

Figure 1. Schematic representation of displays in Experiment 1. Top panel: first frame (left) and last frame (right) of an eightobject display with a horizontal configuration. Bottom left: last frame of a two-object display with a horizontal configuration. Bottom right: last frame of an eight-object display with a vertical configuration. Actual displays were white-on-black with gray boundary lines.

experimental confounds and optimizing the probability that observers would base their judgments on optical TTC rather than on other unreliable cues. First, the spatiotemporal parameters of the virtual objects were selected in such a way that the objects did not overlap on the screen. That is, as the objects approached, they did not occlude one another or exchange positions in the projection plane, which would have made it difficult for observers to identify the objects' display locations.

Second, we attempted to control the scenes so that optical TTC was the only reliable cue to accurate performance (see Tresilian, 1995). For example, the objects' virtual sizes, distances, and speeds were chosen so that veridical information about relative TTC was always provided by optical TTC but not always by relative size, rate of expansion, or by which object would have reached the edge of the clipping plane first (i.e., which object would have begun to disappear from the screen first had the motion continued). ${ }^{6}$

Specifically, we constrained the scenes so that the FA did not always have the largest projected size on the first or the last frame of the scenes; nor did it always have the largest rate of optical expansion. Rather, we created two sets of scenes to control for potential effects of pictorial relative size on judgments of relative TTC (Delucia, 1991a, 1991b). In half of the scenes, pictorial relativesize information suggested that the NA was closer than the FA even though the latter had a smaller TTC. That is, the projected size of the NA on the first and last frames, and its rate of expansion, was either smaller (Set 1) or larger (Set 2) than that of the FA; in the latter, pictorial relative size information was not consistent with relative TTC information. Our attempt to control size-arrival effects focused on the FA and the NA because these were the only objects present in the two-object displays. The other displays contained the FA and the NA in addition to other objects.
Similarly, the spatiotemporal parameters of the virtual objects were selected so that the FA would have reached the edge of the clipping plane first in half of the scenes; in the remaining scenes, the NA would have reached the edge first. To achieve this and to have objects that would have reached the edge first appear in each of the eight display locations, the objects were arranged in either a "horizontal" or "vertical" configuration. In the former, the objects were spread out more along the horizontal axis than along the vertical axis; the converse was true for the vertical configuration. ${ }^{7}$ We note that the display was terminated before any object reached the edge of the clipping plane.

Despite the numerous constraints on the scenes, optical TTC provided veridical information about the relative TTC of the objects.

Table 1

Parameters of a Representative Scene From Experiment 1:

The Visual Angles Subtended by the Objects' Diagonals on the First (VA1) and Last (VA2) Frames in Degrees, and Local and Global Tau

\begin{tabular}{|c|c|c|c|c|c|c|c|c|}
\hline \multirow[b]{2}{*}{ Object } & \multicolumn{4}{|c|}{ Set 1} & \multicolumn{4}{|c|}{ Set 2} \\
\hline & VA1 & VA2 2 & Local & Global & VAI & VA2 & Local & Global \\
\hline FA & 87 & 3.85 & .73 & .72 & .61 & 2.67 & .73 & .72 \\
\hline $\mathrm{NA}$ & .73 & 2.63 & .95 & .92 & 1.05 & 3.81 & .94 & .92 \\
\hline 3. & 1.21 & 3.87 & 1.13 & 1.12 & .84 & 2.68 & 1.13 & 1.11 \\
\hline 4. & .95 & 2.71 & 1.34 & 1.32 & 1.36 & 3.88 & 1.34 & 1.32 \\
\hline 5. & 1.51 & 3.98 & 1.52 & 1.51 & 1.03 & 2.72 & 1.51 & 1.52 \\
\hline 6. & 1.13 & 2.73 & 1.75 & 1.72 & 1.64 & 3.96 & 1.75 & 1.72 \\
\hline 7. & 1.73 & 3.95 & 1.93 & 1.92 & 1.20 & 2.74 & 1.93 & 1.91 \\
\hline 8. & 1.27 & 2.76 & 2.11 & 2.11 & 1.85 & 4.02 & 2.11 & 2.11 \\
\hline
\end{tabular}

Note-FA, first arriving; NA, next arriving. 
To verify this, we estimated local and global tau (Kaiser \& Mowafy, 1993; Tresilian, 1991). Local tau is defined as $\mathrm{A} / \dot{\mathrm{A}} ; \mathrm{A}$ is the angular extent of the object's image (we used the object's diagonal) and $\dot{A}$ is the rate of expansion or temporal derivative of that angle (Kaiser \& Mowafy, 1993; Tresilian, 1991). We refer to the magnitude of change in $\mathrm{A}$ as local expansion. Global tau is defined as $\mathrm{B} / \dot{\mathrm{B}} ; \mathrm{B}$ is the angular extent between an object (we used the center of the object) and an observer's principal line of sight (or track vector if the observer is moving), and $\dot{B}$ is the rate of expansion or temporal derivative of that angle (Kaiser \& Mowafy, 1993; Tresilian, 1991). We refer to the magnitude of change in $\mathrm{B}$ as global expansion. Both forms of tau provided veridical information about relative TTC (e.g., see Table 1).

A "3-2-1" countdown was shown at the beginning of each trial to help observers anticipate the onset of motion. The observers were instructed to fixate the numbers until they disappeared, but eye movements were not recorded. Lines that divided the display into eight areas were present during this countdown and throughout the trial. We did not instruct observers to maintain fixation during approach because it has been reported that observers have difficulty fixating a stable point while attending a moving background during a visual-search task (Khurana \& Kowler, 1987).

Procedure and Design. The observers viewed the displays in a dimly lit room and were instructed to report the location of the object that they thought would "hit" them (or pass them; i.e., reach the observation plane) first had the motion continued at the same speed beyond the screen. They were instructed to press a mouse button as soon as they knew which object would hit them first. Immediately thereafter, they verbally reported the display area in which they thought the object was located by reporting the associated numeral (see also Previc \& Blume, 1993). The observers were instructed to respond as quickly and as confidently as possible.

Feedback was provided in practice trials to help observers learn the task; such trials were not included in data analyses. However, only 32 practice trials were included, and feedback was not provided in experimental trials because we did not want observers to develop artificial response strategies that were not based on their perception of the relative TTC of the objects (as seemed to occur in a control experiment with feedback on experimental trials). Further, we aimed to avoid extremely low error rates, which can lead to limits in the interpretation of RT data (Pachella, 1974). However, we did inform observers that the FA might or might not be the largest object or the object that would leave the screen first - that is, that these cues were not reliable. We note that although Todd (1981) provided feedback, observers reported that they did not use it on many trials.

The trial was terminated upon keypress, and RT and accuracy were recorded. The RT was defined as the time between the presentation of the scene and the observer's keypress. Trials in which there was no response or in which RT was longer than $5 \mathrm{sec}$ were omitted. There were 64 unique scenes, each presented six times. To minimize fatigue, observers completed 192 trials in each of two sessions completed on 2 successive days. Trials were run in a different random order in each session, and the results of an analysis of variance (ANOVA) indicated that effects of session were negligible.

\section{Results and Discussion}

Results are summarized in Figures 2 and 3.

Performance compared with chance. Two-tailed $t$ tests indicated that accuracy was above chance probability in each condition of set size and set $(p<.006 ; d f$ is 7 for all), except with two objects in Set 2 . The finding that observers can judge the relative TTC of as many as eight approaching objects is noteworthy and consistent with prior results demonstrating that observers are highly accurate at detecting small differences in the TTC of two approaching objects (Todd, 1981). However, our observers did not typically achieve the superlative accuracy rates reported by Todd (1981)-even with two-object displays in Set 1 (except when the FA was located in areas 4, 6, 7, and 8; see below) ${ }^{8}$

Our results suggest further that misleading relative size information can result in degraded performanceaccuracy that is not different from chance probabilitywhen only two objects are present. This is consistent with previously reported size-arrival effects (see, e.g., DeLucia, 1991a, 1991b; DeLucia \& Warren, 1994). However, accuracy was above chance with more than two objects in Set 2; the presence of a misleading relative size cue did not degrade performance in these cases. Effects of set may have been attenuated when displays contained more than two objects as a consequence of the previously discussed constraints we imposed on the scenes. For example, with four-, six-, and eight-object displays, neither the FA nor the NA had the largest optical size on the first or last frames. In addition, on the last frame, half of the objects were comparable to one another in optical size and were larger than the other half of the objects, which were also comparable to one another; see Table 1. However, on both the first and last frames of two-object displays, the FA projected the largest image in Set 1; the NA projected the largest image in Set 2.

Analyses of variance. To evaluate performance as a function of set size, we conducted $2 \times 4 \times 8$ (set $\times$ set size $\times$ FA location) repeated measures ANOVAs. The comparison of performance across different levels of set size raised several issues. First, the probability of making a correct response by chance alone (e.g., guessing) decreases as set size increases. Thus, percentage correct is not a suitable measure of effects of set size on performance. Therefore, we converted percentage correct scores to $d^{\prime}$, which measures sensitivity independently of decision criterion (Green \& Swets, 1966) and can be compared across different numbers of alternatives or set sizes (Green \& Swets, 1966; McNichol, 1972; see also Pollack, 1959, 1964). ${ }^{9}$ Our task is analogous to an $m$-alternative forcedchoice complete identification task (Graham, 1989; Luce, Bush, \& Galanter, 1963). Sensitivity was estimated by converting percentage correct scores to $d^{\prime}$ values with $m$-alternative forced-choice tables (Hacker \& Ratcliff, 1979, which is a revision of Elliot, 1964). ${ }^{10}$

The analysis of RT raised additional issues because we had to consider whether to include both correct and incorrect responses or to analyze only correct responses. The removal of errors in analyses of RT is ubiquitous (Pachella, 1974). However, Pachella has convincingly argued that this method is based on questionable assumptions and can lead to incorrect interpretations of RT. To summarize Pachella's most germane points, RT measures from correct responses are typically based on simple tasks that probably could be done with $100 \%$ accuracy if the task was not timed; also, error rates are typically low $(0 \%-10 \%)$. At such rates, even a small change in accuracy that occurs when the independent variable is manipulated can produce a large change in RT. Thus, small differences in errors between experimental conditions 

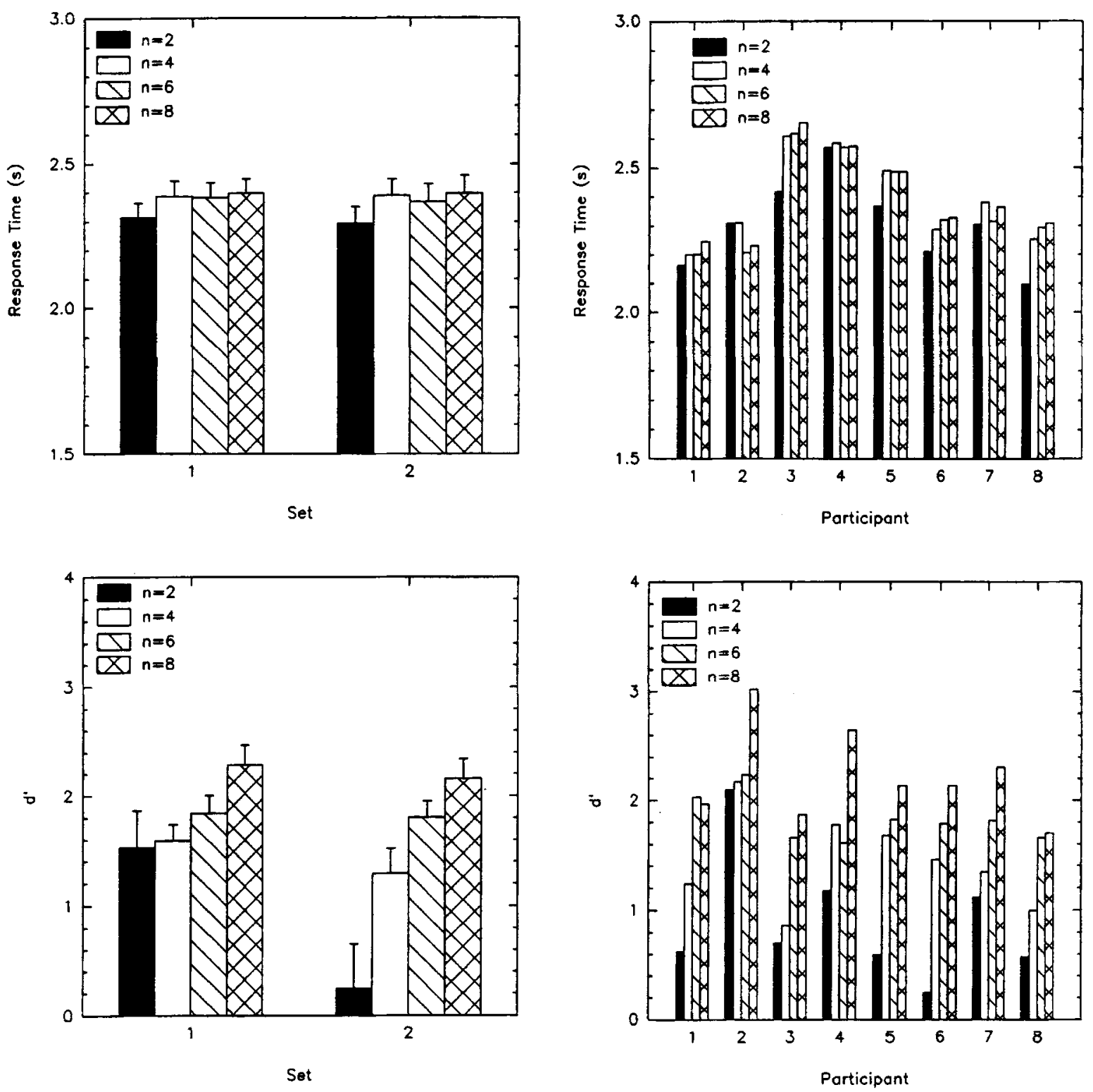

Figure 2. Experiment 1: performance as a function of set size ( $n$ ) and set. Top left: mean response time (RT). Top right: mean RT of individual observers averaged across set and first-arriving object (FA) location. Bottom left: mean $d^{\prime}$. Bottom right: mean $d^{\prime}$ of individual observers averaged across set and FA location. Error bars indicate 1 SEM.

may reflect changes in the observer's speed-accuracy criterion, rather than changes in processes presumed to underlie the task, and thus can lead to potentially misleading interpretations of RT.

Our study does not conform to a traditional RT paradigm. Specifically, the task was relatively complex; displays contained motion, and misleading visual information about relative depth was systematically presented. Further, although observers were instructed to respond as soon as possible, they generally viewed most of the scene before they responded (as indicated by overall mean RT). More important, preliminary analyses of performance in Experiment 1 indicated that observers achieved much higher levels of accuracy when the FA was located in the areas referred to as $4,6,7$, and 8 (overall mean was $93.5 \%$ ) than when the FA was located in the areas referred to as $1,2,3$, and 5 (overall mean was $30.9 \%$ ). Thus, when incorrect responses are removed, those trials in which the FA was located in areas $4,6,7$, and 8 are represented more than the other trials. This could result in a biased measure of RT.

We therefore included an analysis of RT consisting of both incorrect and correct responses. One concern with such an analysis is that incorrect responses may reflect activities unrelated to the processes that underlie relative TTC judgments or may indicate that observers did something unusual on those trials (see Pachella, 1974). Moreover, RT may be greater on incorrect trials than on correct 

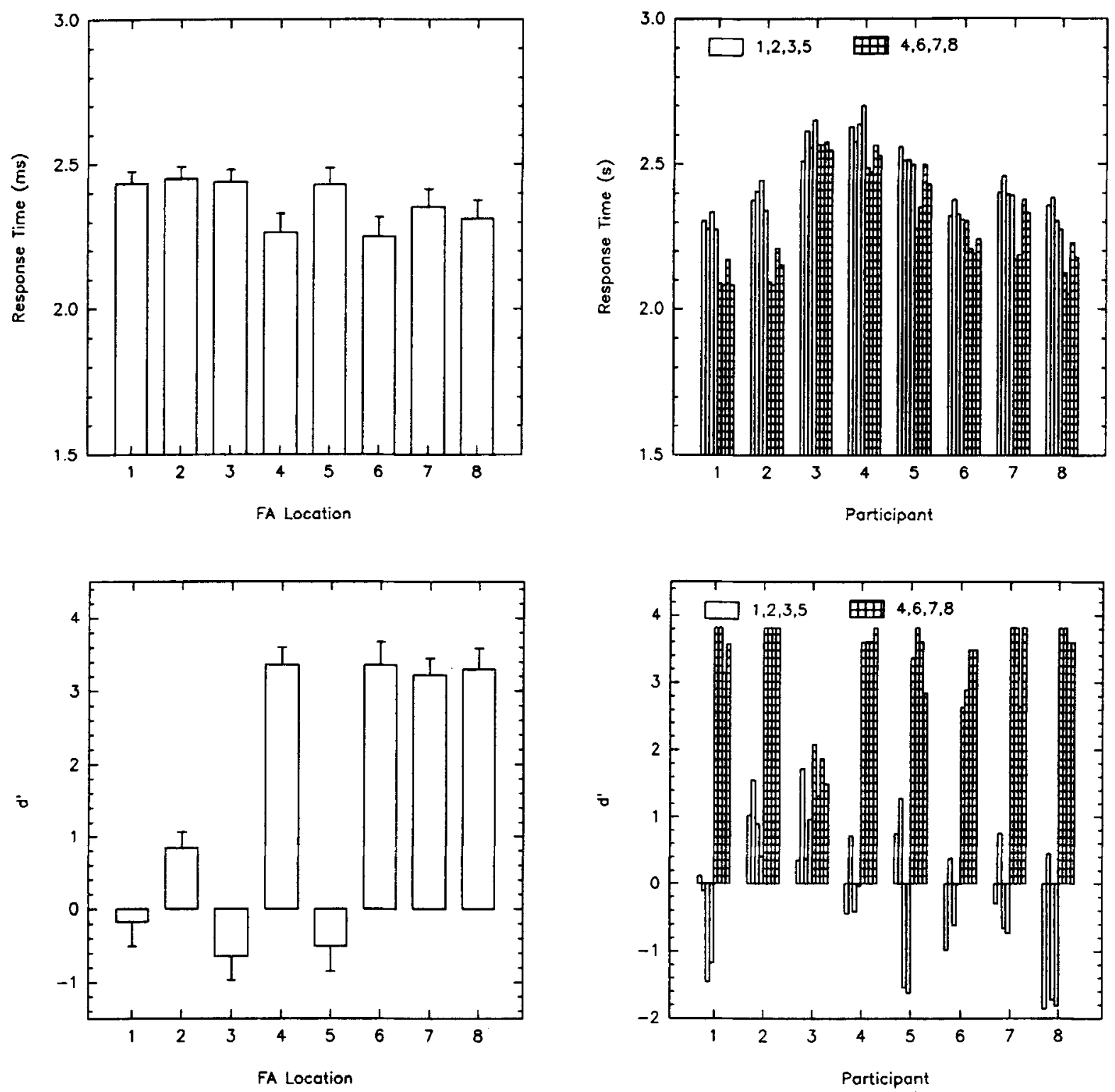

Figure 3. Experiment 1: performance as a function of FA location. Top left: mean response time (RT). Top right: mean RT of individual observers. Bottom left: mean $d^{\prime}$. Bottom right: mean $d^{\prime}$ of individual observers. Error bars indicate $1 S E M$.

trials, and more incorrect responses may occur with larger set sizes. This would lead to greater RT with larger set sizes due to a disproportionate contribution of incorrect responses to the larger set sizes. Consequently, if processes that underlie incorrect responses differ from those that underlie correct responses, the conclusion that greater RT occurs with larger set sizes because of limited-capacity processing would be unwarranted.

To address this concern, we computed the mean RT of correct responses separately from the mean RT of incorrect responses for each observer in each condition of set and set size, averaged across FA location (and collapsed across replications; as noted earlier, the number of cor- rect and incorrect responses was not the same for all conditions and observers). We then computed the difference between the mean RT of correct and incorrect responses. We subjected these differences to a repeated measures ANOVA to evaluate whether they varied with set size. There were no effects of set size in Experiment 1 (or 2). This ameliorated our concern that the inclusion of incorrect trials may lead to unwarranted interpretations of greater mean RTs with larger set sizes. Therefore, notwithstanding the caveats noted above, we included correct and incorrect responses in our analyses of RT.

Mean RT. There was a main effect of set size on RT $\left[F(3,21)=5.75, M S_{\mathrm{e}}=.04, p<.0389, w^{2}=2.89 \%\right]{ }^{.11}$ 
Results of Tukey's HSD tests indicated that mean RT was greater with four, six, or eight objects than with two ( $p<$ .05 ; means were $2.30,2.38,2.39$, and $2.40 \mathrm{sec}){ }^{12}$

Mean $d^{\prime}$. There was a main effect of set size on $d^{\prime}$ $\left[F(3,21)=39.14, M S_{\mathrm{e}}=1.052, p<.0001, w^{2}=4.80 \%\right]^{13}$ and its interaction with set $\left[F(3,21)=5.88, M S_{\mathrm{e}}=1.791\right.$, $p<.0349, w^{2}=1.05 \%$ ]. Simple main effects indicated that in Set $1, d^{\prime}$ was greater with eight objects than with two or four objects $(p<.05)$. In Set $2, d^{\prime}$ was greater with four, six, and eight objects than with two objects, and was greater with eight objects than with four objects $(p<.01)$.

Ratio of $d^{\prime} / \mathbf{R T}$. The above results suggest a speedaccuracy tradeoff; both RT and $d^{\prime}$ (an accuracy measure, Townsend \& Ashby, 1983) were generally smaller with two-object displays than with larger set sizes. Thus, we also analyzed the ratio of $d^{\prime}$ to RT (see also Cheal \& Lyon, 1992; Townsend \& Ashby, 1983). Results again indicated a main effect of set size $\left[F(3,21)=39.74, M S_{\mathrm{e}}=\right.$ $\left..181, p<.0001, w^{2}=4.31 \%\right]$. The ratio increased as set size increased; all pairwise comparisons were significant $(p<.05)$. There also was an effect of FA location $[F(7,49)$ $=43.88, M S_{\mathrm{e}}=1.023, p<.0001, w^{2}=62.84 \%$; the pattern of results was as described below], and an interaction between set size and set $\left[F(3,21)=6.47, M S_{\mathrm{e}}=\right.$ $\left..3389, p<.0308, w^{2}=1.14 \%\right]$. Simple main effects indicated the same pattern as with $d^{\prime} .{ }^{14}$

Effects of FA location on performance. The location of the FA varied among the eight display locations from trial to trial to ensure that observers could not anticipate FA location. We also eliminated confounds between FA location and set size by placing FA in each of the eight display areas an equal number of times in each condition of set size. Effects of FA location were not anticipated. However, an analysis of such effects potentially provides information about what visual information observers used to perform the task. A summary of the most relevant results of the analyses of RT and $d^{\prime}$ is presented below; see also Figure 3.

There was a main effect of FA location on $\operatorname{RT}[F(7,49)=$ $\left.16.23, M S_{\mathrm{e}}=.03, p<.0001, w^{2}=14.07 \%\right]$ and $d^{\prime}$ $\left[F(7,49)=46.10, M S_{\mathrm{e}}=4.93, p<.0001, w^{2}=62.09 \%\right]$. There was also an interactive effect of set and FA location on $\operatorname{RT}\left[F(7,49)=3.54, M S_{\mathrm{e}}=.01, p<.0359, w^{2}=\right.$ $.76 \%$ ]. In general, performance was typically better with scenes in which the FA was located in the areas referred to as $4,6,7$, and 8 than with FA locations $1,2,3$, and 5 . When averaged across set, set size and FA locations 4,6 , 7 , and $8, d^{\prime}$ was 3.31 . When averaged across FA locations $1,2,3$, and $5, d^{\prime}$ was -.12 . Corresponding means for RT were 2.29 and $2.44 \mathrm{sec}$. The same pattern of results regarding FA location occurred with all set sizes.

We attempted to determine the basis of the effects of FA location by analyzing differences in the displays between FA locations 4, 6, 7, and 8 and FA locations 1, 2, 3, and 5 . In the former scenes, the FA would have reached the edge of the clipping plane first. Such scenes resulted in relatively better performance. In the latter scenes, the NA would have reached the edge first and performance was relatively worse. This difference in performance be- tween the two groups of scenes suggests that this "edge cue" contributed to observers' judgments (see Law et al., 1993, for a similar approach). We note that the display was terminated before any object reached the edge, and that the effect of FA location occurred even when feedback was provided in a control experiment.

We examined effects of FA location further with analyses of the optical properties of the displays. Local and global expansion increase as the distance between an approaching object and the observation plane decreases at a constant rate (see, e.g., Hochberg, 1986). ${ }^{15}$ However, local expansion or global expansion alone does not unambiguously specify TTC; for example, two objects with the same TTC can have different rates of expansion. We considered the possibility that effects of FA location on performance occurred because observers relied, at least in part, on lower order information of local or global expansion, which may have conflicted with relative TTC information. Specifically, we determined which of the eight objects produced the largest local expansion and the largest global expansion in each of the conditions of FA location and set (computations were based on the entire scene duration).

In Set 1, when FA was located in areas 1, 2, 3, or 5, the FA had the largest local expansion and the NA had the largest global expansion (Table 2). In this case, the objects' relative magnitudes of global expansion conflicted with their relative TTC information. In Set 2, NA had the largest local and global expansion; both conflicted with their relative TTC information. However, when FA was located in areas $4,6,7$, or 8 , FA had the largest local expansion and global expansion in Set 1 , consistent with relative TTC information. In Set 2, the FA had the largest global expansion, but the NA had the largest local expansion. In this case, the objects' relative magnitudes of local expansion conflicted with relative TTC information; however, their relative magnitudes of global expansion were consistent with relative TTC information. Thus, performance was less effective when global expansion contradicted TTC information than when local expansion contradicted TTC information, which suggests that global expansion is relatively more effective in relative TTC judgments. Further, a comparison of scenes in which the FA was located in areas $1,2,3$, and 5 with the remaining scenes indicated that, in the latter, the FA's global expansion was larger and the ratio of local expansion to global expansion was smaller; the reverse was true for the NA.

We note that when the FA or the NA had the largest global expansion, it also had the largest optical extent between the object and the principal line of sight on the last frame (and also on the first frame in some cases). Further study is needed to differentiate the contributions of various global and local optical properties. Nevertheless, our results suggest that observers relied on cues other than, or in conjunction with, optical TTC to make relative TTC judgments, which is consistent with prior studies (see, e.g., DeLucia, 1991a, 199 1b; Kaiser \& Mowafy, 1993; Law et al., 1993). 
Table 2

Magnitude of Local and Global Optical Expansion (in Degrees, and Based on the Entire Scene Duration of $2.48 \mathrm{sec}$ ) and Local and Global Tau, of First-Arriving (FA) and Next-Arriving (NA) Objects as a Function of the Eight Scenes in Which FA Display Locations Varied

\begin{tabular}{|c|c|c|c|c|c|c|c|c|}
\hline \multirow{2}{*}{$\begin{array}{c}\text { FA } \\
\text { Location } \\
\end{array}$} & \multicolumn{2}{|c|}{ Local Expansion } & \multicolumn{2}{|c|}{ Global Expansion } & \multicolumn{2}{|c|}{ Local Tau } & \multicolumn{2}{|c|}{ Global Tau } \\
\hline & FA & NA & FA & NA & FA & NA & FA & NA \\
\hline \multicolumn{9}{|c|}{ Set 1} \\
\hline 1. & 2.98 & 1.90 & 6.94 & 10.59 & .73 & .95 & .72 & .92 \\
\hline 2. & 2.98 & 1.90 & 6.94 & 10.59 & .73 & .95 & .72 & .92 \\
\hline 3. & 2.99 & 1.93 & 6.46 & 8.16 & .73 & .93 & .72 & .92 \\
\hline 4. & 2.91 & 1.95 & 11.82 & 6.21 & .74 & .93 & .72 & .92 \\
\hline$\overline{5}$ & 2.99 & 1.93 & 6.46 & 8.16 & .73 & .93 & .72 & .92 \\
\hline$\underline{6}$. & 2.91 & 1.95 & 11.82 & 6.21 & .74 & .93 & .72 & .92 \\
\hline ‥ & 2.96 & 1.96 & 9.12 & 5.78 & .73 & .92 & .72 & .92 \\
\hline$\underline{8}$. & 2.96 & 1.96 & 9.12 & 5.78 & .73 & .92 & .72 & .92 \\
\hline \multicolumn{9}{|c|}{ Set 2} \\
\hline 1. & 2.06 & 2.76 & 7.76 & 10.06 & .73 & .94 & .72 & .92 \\
\hline 2. & 2.06 & 2.75 & 7.76 & 10.06 & .73 & .96 & .72 & .92 \\
\hline 3. & 2.07 & 2.79 & 7.22 & 7.76 & .73 & .94 & .72 & .92 \\
\hline$\underline{4}$. & 1.99 & 2.81 & 13.21 & 5.90 & .75 & .94 & .72 & .92 \\
\hline 5. & 2.07 & 2.79 & 7.23 & 7.76 & .73 & .94 & .72 & .92 \\
\hline$\underline{6}$. & 1.99 & 2.81 & 13.21 & 5.90 & .75 & .94 & .72 & .92 \\
\hline 7. & 2.04 & 2.82 & 10.19 & 5.49 & .74 & .93 & .72 & .92 \\
\hline$\underline{8}$. & 2.04 & 2.82 & 10.19 & 5.49 & .74 & .93 & .72 & .92 \\
\hline
\end{tabular}

Note-Underlined numerals indicate scenes with better performance.

Analysis of errors. We also determined which objects were selected when the FA was not selected. A summary of such response errors is shown in Figure 4. Note that with two-object displays, the NA is the only alternative to the FA; this condition is not informative. We limit discussion to set sizes greater than two; the object that was selected most frequently did not vary across set size except for a tie in one condition.

An analysis of all scenes combined indicated that when observers responded incorrectly, they selected the NA most frequently. When scenes in which the FA was located in areas 1, 2, 3, and 5 were analyzed separately from scenes in which the FA was located in areas $4,6,7$, and 8 , results indicated that the NA was selected most frequently, except with the latter scenes in Set 1 ; in this case, the object that was selected most frequently had the third largest TTC (with six-object displays, there was a tie between objects with third and fifth largest TTCs). However, when the FA was located in areas 1, 2, 3, and 5, the number of errors ranged from 118 to 151 across conditions of set size and set; in the remaining scenes, the range was 5 to 14 . Thus, we interpret errors from the latter scenes with caution.

It is reasonable to propose that because the NA was typically selected most frequently, observers based their judgments on optical TTC. In other words, perhaps the difference in TTC between the FA and the NA was below threshold despite our use of a 200-msec differential; further, information that contradicts relative TTC (e.g., global expansion) may raise the threshold. Alternatively, the NA may have been selected due to other optical properties, contingently related to TTC, which may underlie the pattern of errors. Thus, we compared several optical prop- erties of the incorrectly selected objects with those of all other objects (except the FA). We report those cases in which an object had the largest parameter in all scenes (i.e., scenes that differed in FA location) unless otherwise noted. When the FA was located in areas 1,2,3, and 5 (in Sets 1 and 2), NA had the largest global expansion; it also had the largest optical extent between the object and the principal line of sight on the last frame (and the first frame, with two exceptions; in Set 2, the NA also had the largest local expansion). This is consistent with our prior analyses, which suggest that global optical variables contribute to observers' judgments. With FA locations 4, 6,7 , and 8 and Set 2, the NA had the largest local and global expansion except with FA location 4 (in this case, the object with the fourth smallest TTC had the largest global expansion, but this scene did not produce selections of this object). In Set 1 , the object with the third smallest TTC was selected most frequently (except with the tie noted earlier) and had the largest local expansion.

In sum, the analyses of response errors and effects of FA location suggest that information other than optical TTC contributes to relative TTC judgments. Global optical variables seem particularly effective, but a more systematic manipulation of local and global optical variables is warranted.

Efficiency. The finding that accuracy was above chance in all conditions except with two-object displays in Set 2 suggests that misleading visual information may be more effective with smaller set sizes. A general implication is that the number of sources of visual information and the type of visual information that affects relative TTC judgments may vary with set size. That is, an observer's ability to use multiple sources of visual information (regardless 

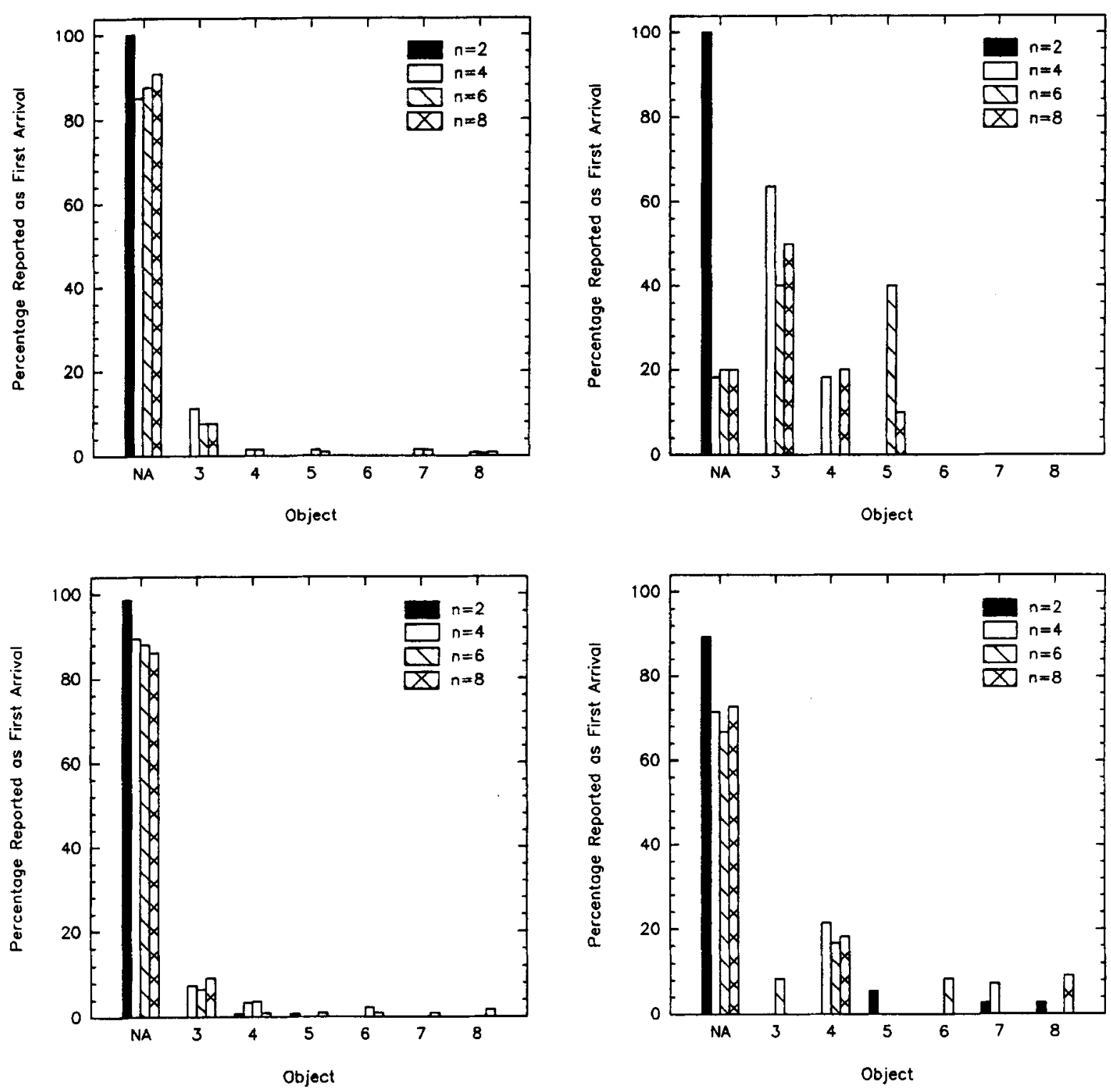

Figure 4. Experiment 1: percentage of error trials in which an object other than the first-arriving object (FA) was reported as the first to arrive. Object number indicates rank of time-to-contact (TTC; $3=$ object with third smallest TTC). Set size is shown in ascending order from left to right for each value on the horizontal axis (shading for six and eight objects does not appear with small percentages), and objects with values greater than a given set size refer to empty display locations (it is not clear why observers selected these on some trials, particularly in Set 2 with FA locations 4, 6, 7, and 8). Top left: FA locations 1, 2, 3, and 5, and Set 1. Top right: FA locations 4, 6, 7, and 8, and Set 1. Bottom left: FA locations 1, 2, 3, and 5, and Set 2. Bottom right: FA locations $4,6,7$, and 8 , and Set 2 .

of whether those sources are veridical) in judgments of relative TTC may be greater with smaller set sizes. As set size increases, the number of sources of visual information that observers can use may decrease; as a consequence, the effectiveness of misleading information may also decrease. This is consistent with the aforementioned finding (notwithstanding the previously discussed possibility that this was due to constraints in scene design).

To further explore this possibility, we analyzed a statistical efficiency measure of performance (Barlow, 1978). We computed the ratio of $d^{\prime}$ obtained by each observer in each condition of set size, set, and FA location to that achieved by an ideal observer. For the purpose of this analysis, we assumed that the latter would achieve $100 \%$ accuracy in all conditions and thus used the maximum (positive) $d^{\prime}$ in the corresponding condition as the ideal score. We then squared this ratio to get a statistical efficiency score (see Barlow, 1978). ${ }^{16}$ In our previous analyses, we interpreted $d^{\prime}$ as an index of sensitivity and retained the sign of the scores (positive $d^{\prime}$ scores reflected greater sensitivity than did negative $d^{\prime}$ scores). In the efficiency analysis, negative $d^{\prime}$ values result in positive ef- 
Table 3

Parameters of a Representative Scene in Experiment 2: The Visual Angles Subtended by the Objects' Diagonals on the First (VA1) and Last (VA2) Frames in Degrees, and Local and Global Tau

\begin{tabular}{|c|c|c|c|c|c|c|c|c|}
\hline \multirow[b]{2}{*}{ Object } & \multicolumn{4}{|c|}{ Set 1} & \multicolumn{4}{|c|}{ Set 2} \\
\hline & VAl & VA2 & Local & Global & VA1 & VA2 & Local & Global \\
\hline \multicolumn{9}{|c|}{ Target Present } \\
\hline $\mathrm{FA}$ & .91 & 4.01 & .73 & .72 & .62 & 2.73 & .73 & .72 \\
\hline 2. & .86 & 2.73 & 1.15 & 1.12 & 1.26 & 4.00 & 1.14 & 1.11 \\
\hline 3. & 1.27 & 4.05 & 1.13 & 1.12 & .86 & 2.77 & 1.13 & 1.11 \\
\hline 4. & .84 & 2.66 & 1.14 & 1.12 & 1.26 & 3.99 & 1.14 & 1.12 \\
\hline 5. & 1.26 & 4.04 & 1.12 & 1.1 & .87 & 2.81 & 1.12 & 1.11 \\
\hline 6. & .87 & 2.78 & 1.13 & 1.11 & 1.26 & 4.02 & 1.13 & 1.12 \\
\hline 7. & 1.26 & 4.02 & 1.13 & 1.12 & .87 & 2.77 & 1.14 & 1.12 \\
\hline 8. & .87 & 2.79 & 1.12 & 1.11 & 1.26 & 4.06 & 1.12 & 1.11 \\
\hline \multicolumn{9}{|c|}{ Target Absent } \\
\hline 1. & 1.26 & 4.05 & 1.12 & 1.12 & .87 & 2.78 & 1.13 & 1.12 \\
\hline 2. & .86 & 2.73 & 1.15 & 1.12 & 1.26 & 4.00 & 1.14 & 1.11 \\
\hline 3. & 1.27 & 4.05 & 1.13 & 1.12 & .86 & 2.77 & 1.13 & 1.11 \\
\hline 4. & .84 & 2.66 & 1.14 & 1.12 & 1.26 & 3.99 & 1.14 & 1.12 \\
\hline 5. & 1.26 & 4.04 & 1.12 & 1.11 & .87 & 2.81 & 1.12 & 1.11 \\
\hline 6. & .87 & 2.78 & 1.13 & 1.11 & 1.26 & 4.02 & 1.13 & 1.12 \\
\hline 7. & 1.26 & 4.02 & 1.13 & 1.12 & .87 & 2.77 & 1.14 & 1.12 \\
\hline 8. & .87 & 2.79 & 1.12 & 1.11 & 1.26 & 4.06 & 1.12 & 1.11 \\
\hline
\end{tabular}

ficiency scores (due to the squaring of the ratio). We note that negative $d^{\prime}$ values indicate that observers performed reliably below chance and thus avoided the correct alternative systematically (see Macmillan \& Creelman, 1991) and thus may have relied on unreliable visual information systematically. Thus, in the efficiency analysis, we assume that $d^{\prime}$ values that deviate from 0 (chance) in either direction represent the systematic use of visual information, which is another index of performance.

Results indicated that there was a main effect of set size $\left[F(3,21)=6.51, M S_{\mathrm{e}}=.09, p<.0222, w^{2}=1.52 \%\right]$. In contrast to analyses of $d^{\prime}$, mean efficiency was greater with two objects than with the other set sizes $(p<.05$; means were $.48, .48, .49$, and .62 ). There were also interactions between set size and set $\left[F(3,21)=7.76, M S_{\mathrm{e}}=\right.$ $\left..02, p<.0055, w^{2}=.51 \%\right]$ and between set size and FA location $\left[F(21,147)=2.79, M S_{\mathrm{e}}=.06, p<.0376, w^{2}=\right.$ $2.03 \%$ ]; further study is warranted. The pattern of results regarding FA location was similar to that obtained with $d^{\prime}$. Results suggest that with smaller set sizes, observers may be relatively more efficient at using more sources of visual information, but misleading information may also be more effective with smaller set sizes.

\section{EXPERIMENT 2}

In Experiment 1, observers performed an identification task that closely resembles relative TTC judgments measured in earlier studies. In Experiment 2, observers performed a detection task; they judged whether a target (an object with a TTC that differed from that of the other objects) was present or absent among a variable number of distractors.

\section{Method}

Observers. Eight observers had the same characteristics as those in Experiment 1 except that all received course credit.

Displays and Procedure. Displays were generally similar to those in Experiment 1. Optical parameters are shown in Table 3. Two, four, six, or eight objects approached the observer. However, on half of the trials, one of the objects had a TTC value of .7 sec on the last frame; the remaining objects had a TTC of $1.1 \mathrm{sec}$. On these trials, only one object, the target, would have hit the observation point before the others; the latter would have arrived at the same time as each other. On the remaining trials, all objects would have arrived at the same time. The observers were instructed to report whether all objects would hit them at the same time or whether one object would arrive sooner than the others; that is, they were instructed to report whether a target was present (an object that would arrive sooner) or absent (all objects would arrive at the same time) by pressing one of two mouse buttons. The chance probability of a correct response was the same regardless of set size.

All aspects of the displays were not identical to those in Experiment 1 . In the latter, one object (FA) would have reached the edge first when it was located in areas $4,6,7$, and 8 , and another object (NA) would have reached the edge first in the remaining scenes. In Experiment 2, this pattern occurred only when set size was two and a target was absent (in such target-absent scenes, all objects arrived at the same time; no object is designated as the FA or the NA). In remaining target-absent scenes, one or more of the other objects would have reached the edge first. In target-present scenes, the FA or the FA and one or more other objects would have reached the edge first.

\section{Results and Discussion}

Results are shown in Figure 5.

Performance compared with chance. Accuracy was above chance probability for all levels of set size and set $(p<.006)$.

Mean RT. Results of a $2 \times 4 \times 8$ repeated measures ANOVA (set $\times$ set size $\times$ FA location) indicated a main effect of set size on RT $\left[F(3,21)=6.68, M S_{\mathrm{e}}=.02, p<\right.$ $.0039]$, but it accounted for only $.62 \%$ of the variance. Unlike the RT in Experiment 1, RT was faster with eight objects $(2.38 \mathrm{sec})$ than with two $(2.45 \mathrm{sec})$, four $(2.45 \mathrm{sec})$, or six $(2.44 \mathrm{sec})$ objects $(p<.05)$.

Mean $\boldsymbol{d}^{\prime}$. The task in Experiment 2 can be considered a yes - no detection task in a signal detection theory framework. Thus, we computed the proportion of hits and false alarms for each observer in each combination of set, set size, and FA location. We then converted these proportions to $d$ ' values with Elliot's (1964) yes-no tables. There was an effect of set size $\left[F(3,21)=6.16, M S_{\mathrm{e}}=3.72, p<\right.$ $.0153, w^{2}=3.32 \%$ ]. Sensitivity was greater with six or eight objects than with two $(p<.05)$. Also, mean $d^{\prime}$ was greater in Set 2 than in Set $1\left[F(1,7)=8.89, M S_{\mathrm{e}}=2.36\right.$, $\left.p<.0204, w^{2}=1.07 \%\right]$; further investigation is needed to explain this result. Finally, the ratio of $d^{\prime}$ to RT was greater $(p<.05)$, with a set size of eight or six than with two, was greater with Set 2 than with Set 1 , and was greater with FA locations $4,6,7$, and 8 than with locations 1,2 , 3 , and 5 . There were significant effects of set and FA location, but not set size, on efficiency.

Effects of FA location on performance. There was a main effect of FA location on RT $\left[F(7,49)=5.07, M S_{\mathrm{e}}=\right.$ $\left..02, p<.0046, w^{2}=1.23 \%\right]$ and $d^{\prime}[F(7,49)=15.73$, 

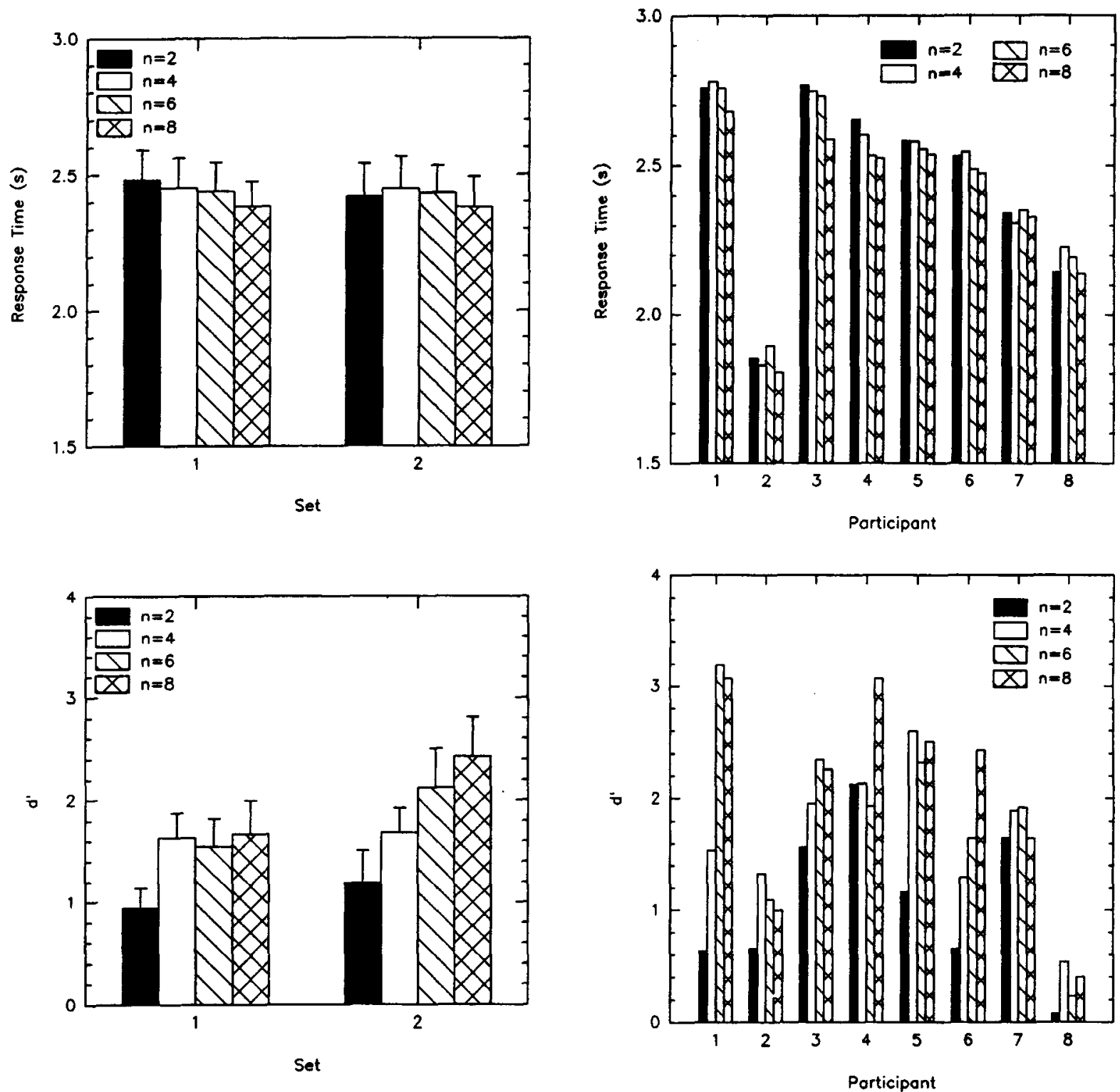

Figure 5. Experiment 2: performance as a function of set size ( $n$ ) and set. Top left: mean response time (RT). Top right: mean RT of individual observers averaged across set and FA location. Bottom left: mean $d^{\prime}$. Bottom right: mean $d^{\prime}$ of individual observers averaged across set and FA location. Error bars indicate $1 S E M$.

$\left.M S_{\mathrm{e}}=3.92, p<.0002, w^{2}=23.24 \%\right]$. As in Experiment 1 , sensitivity was greater and RT was smaller with FA locations $4,6,7$, and 8 than with the remaining scenes. We noted earlier that displays in Experiment 2 were not identical to those in Experiment 1 with respect to the edge cue; thus, the present results suggest that effects of FA location are not due to the edge cue per se.

We again analyzed local and global expansion. We note that the target-present scenes and target-absent scenes were identical except for the spatiotemporal parameters of one object; to create target-absent scenes, the object with a TTC of .7 sec (the FA) was replaced with an object with a TTC of $1.1 \mathrm{sec}$. Thus, we evaluated how the local and global expansion of that one object differed between the four scenes that resulted in the best performance and the four scenes that resulted in the worst performance; we then compared such differences across target-present and target-absent scenes.

Our analysis indicates that the object's magnitude of local expansion ranged from about $2.0^{\circ}$ to $3.1^{\circ}$ in all targetpresent scenes; the range was $1.9^{\circ}$ to $2.8^{\circ}$ in all targetabsent scenes. However, in the four target-present scenes that resulted in the best performance, the magnitude of global expansion ranged from $9.1^{\circ}$ to $13.2^{\circ}$; in the four target-present scenes that resulted in the worst performance, the range was $6.5^{\circ}$ to $7.8^{\circ}$. The corresponding values for target-absent scenes were $5.9^{\circ}$ to $8.5^{\circ}$, and $4.2^{\circ}$ to $5.0^{\circ}$. Thus, in both target-present and target-absent scenes, 
the magnitude and range of global expansion were substantially greater in the scenes that resulted in the best performance than in the scenes that resulted in the worst performance (a similar pattern occurred with the optical extent between the object and line of sight on first and last frames); local expansion was comparable across the scenes. Moreover, whereas the magnitude and range of local expansion were comparable across target-present $\left(2.0^{\circ}-3.1^{\circ}\right)$ and target-absent $\left(1.9^{\circ}-2.8^{\circ}\right)$ scenes, the magnitude and range of global expansion were greater for target-present scenes than for target-absent scenes, especially in those scenes in which performance was most effective (a similar pattern occurred with the optical extent between the object and line of sight on the last frame). This suggests that global expansion was relatively more effective, consistent with results of Experiment 1.

In summary, the results of Experiment 2 indicate that when as many as eight objects are shown, observers can detect whether one object would hit the observation plane sooner than the others with above-chance probability. This capability is consistent with that of the identification task in Experiment 1. However, unlike the mean RT in Experiment 1, mean RT was faster with eight objects than with two, four, or six objects (although $w^{2}$ was less than $1 \%$ ). Such results may indicate that detection performance is less constrained by limits in cognitive processing than is identification performance. We note that the difference in TTC between the FA and the other objects in the target-present scenes in Experiment 2 was greater than the corresponding difference between the FA and the NA in Experiment $1(200 \mathrm{vs} .400 \mathrm{msec})$. The larger difference in Experiment 2 was based on informal observations of various scenes and results of a pilot study; we aimed to avoid floor or ceiling effects with both targetpresent and target-absent scenes. Further study with a wider range of differential TTC values might determine the basis of the differences between the results of Experiments 1 and 2.

Caveats. The following caveat is based on an analysis described by Duncan and Humphreys (1989) within the context of the visual-search paradigm. The approaching objects in Experiment 2 may result in the appearance of a global, overall shape. This shape may differ when the target is present compared with when it is absent. Thus, observers may simply search for the presence of an object that alters the global shape (rather than use TTC information). Such a strategy would seem to be facilitated by the presence of more objects, which would create a more salient global pattern; a global shape might not emerge with too few objects and might account for the faster RT with eight objects compared with the other set sizes. This kind of strategy can be considered a special, degenerate form of search (Duncan \& Humphreys, 1989).

\section{Relationship Between Experiment 2 and the Visual-Search Paradigm}

The objective of Experiment 2 was to measure the effects of set size on a TTC-detection task. We did not design it as a traditional visual-search task (see, e.g., Treis- man \& Gelade, 1980). For example, observers did not get feedback, as is typical in visual-search studies, and a conjunction-search task (discussed below) was not included. Also, as discussed in Experiment 1, the type of visual information that affects relative TTC judgments may vary with set size. However, given the similarities between Experiment 2 and the feature-search task in the visual-search paradigm, it is useful to consider our data within that framework.

Previous studies. In the feature-search task, observers search for a target among a variable number of distractors (set size). A single feature distinguishes the target from the distractors, and it is assumed that if the feature can be detected in parallel, search time will not vary with set size (see, e.g., Treisman \& Gelade, 1980). Parallel search can be characterized by search times of less than $10 \mathrm{msec}$ per element in target-present scenes, estimated from the slope of the function relating set size and RT (of correct responses); also, the ratio of the slope for targetpresent scenes to the slope for target-absent scenes is typically less than .50 (see Treisman \& Gelade, 1980; Treisman \& Gormican, 1988). Alternatively, a serial search is presumed to occur when observers search for a target that is specified by a conjunction of two features (Treisman \& Gelade, 1980). The function relating RT (of correct responses) and set size increases linearly. Conjunction search can be characterized by search times greater than $10 \mathrm{msec}$; also, the ratio of target-present to target-absent slopes approximates .5, interpreted as a selfterminating search (Treisman \& Gormican, 1988).

Prior results are consistent with parallel search for simple features such as color and shape (see, e.g., Treisman \& Gelade, 1980), and also for more complex properties such as the conjunction of form and movement (McLeod, Driver, \& Crisp, 1988) and 3-D orientation from spatial relations between lines (see, e.g., Enns \& Rensink, 1990). In the visual-search study most closely related to ours, Braddick and Holliday (1991; see also Braddick, 1993; Regan, 1993) obtained results that were consistent with serial search for divergence of the optic flow field. Divergence is a transformation of the optic flow associated with objects that approach (optical expansion) or recede (optical contraction) from the observation point. Results indicated that RT increased linearly with set size, and the ratio of target-present to target-absent slopes approximated .50 . However, it is important to note that although the objects expanded optically, other optical properties associated with 3-D approaches were not present in the displays. For example, each object's expansion remained centered around a fixed point; optical displacement of the objects away from the principal line of sight did not occur. In our study, displays provided a more complete simulation of approach motion. Further, the value of TTC rather than the type of optical transformation distinguished the target from the distractors. A parallel search of TTC is consistent with the notion that there are mechanisms that are selectively responsive to optical TTC (for similar logic, see Braddick \& Holliday, 1991). Indeed, on the basis of results from psychophysical methods, Regan 
and Hamstra (1993) concluded that there is a mechanism that is sensitive to the ratio of an object's angular subtense to the object's rate of increase of the angular subtense (one form of optical TTC). Further, Wang and Frost (1992) reported that there are neurons in the pigeon brain that respond maximally at a constant time before a computergenerated approaching stimulus would contact the bird; they proposed that these neurons signal time-to-collision of approaching objects.

Analyses. From the results of Experiment 2, we computed the slope of the relationship between set size and RT (of correct responses). Averaged over set and FA location, we obtained slopes of $11.22 \mathrm{msec}$ for target-present trials; the slope was $-21.16 \mathrm{msec}$ for target-absent trials. In Set 1 alone, the slopes for target-present and targetabsent scenes were $1.75 \mathrm{msec}$ and $-33.25 \mathrm{msec}$, respectively. In Set 2, corresponding slopes were $20.69 \mathrm{msec}$ and $-9.06 \mathrm{msec}$. None of the slopes were significantly different from zero. The slope for target-present scenes in Set 1 is consistent with parallel search. However, the negative slopes for target-absent scenes seem consistent with the degenerate search strategy discussed previously (and may suggest different search strategies for target-present trials and target-absent trials). Further study is required for a more complete comparison of the judgments measured in Experiment 2 with the traditional visual-search task.

\section{GENERAL DISCUSSION}

The primary objective of the present study was to develop a method to measure relative TTC judgments with displays that contained more objects than those used in previous studies. We also began to evaluate how set size affects performance and whether relative TTC judgments are consistent with limited-capacity processing. The results of Experiment 1 indicated that observers can judge which of as many as eight approaching objects would hit them first with accuracy levels that are significantly better than chance probability. Further, $d^{\prime}$ was generally greater with relatively larger set sizes. However, accuracy fell to chance levels when pictorial relative size information contradicted relative TTC information and set size was two, consistent with prior studies (DeLucia, 1991a, 1991b; DeLucia \& Warren, 1994).

Accuracy was above chance when displays contained more than two objects even in Set 2, but not when displays contained two objects in Set 2. Moreover, efficiency was greatest with two-object displays. One potential explanation of such results is that observers may be relatively more efficient at using multiple sources of visual information with smaller set sizes; as a consequence, misleading information may also be more effective with smaller set sizes. More generally, the number of sources of visual information and the type of visual information that affects relative TTC judgments may vary with set size. Similarly, our results may also suggest that observers used veridical information more effectively as set size increased; for example, as the number of objects increases, the number of distances between objects increases and provides more equations for a structure-from-motion solution (Braunstein, personal communication, August 15, 1996). However, further study is needed before such conclusions can be drawn. For example, as discussed previously, the above-chance accuracy obtained with more than two objects in Set 2 could have been due to constraints in our scene design. Also, there was an effect of FA location on $d^{\prime}$ with all set sizes. Thus, we cannot be sure whether the generally greater mean $d^{\prime}$ with larger set sizes reflects the use of veridical information (e.g., optical TTC) or the use of other information that was contingently related to TTC (see also our subsequent discussion of caveats).

In general, mean RT was greater with eight objects than with two, which is consistent with limited-capacity processing. ${ }^{17}$ Thus, even if observers can use optical TTC, capacity limits in memory and attention (see, e.g., Cowan, 1988; Kahneman, 1973; Shiffrin, 1976) may constrain an observer's ability to process information about scenes and thus to judge TTC (see also Law et al., 1993).

Analyses of the optical properties of the displays indicated that performance was less effective when global expansion contradicted TTC information than when local expansion contradicted TTC information. This suggests that observers relied on cues other than, or in conjunction with, optical TTC to make relative TTC judgments, which is consistent with prior studies (see, e.g., DeLucia, 1991a, 1991b; Kaiser \& Mowafy, 1993; Law et al., 1993). Global optical variables seem particularly effective; however, a more systematic manipulation of various local and global optical variables is warranted.

The results of Experiment 2 indicated that RT was faster with eight objects than with the other set sizes. Thus, detection performance may be less constrained by limits in cognitive processing than was the identification task used in Experiment 1 (although differential TTC was smaller in the latter). Moreover, results were consistent with parallel visual search of target-present scenes in Set 1 , but results of target-absent scenes generally seemed consistent with a degenerate search strategy. Future experiments on visual search for TTC that match the traditional visual-search paradigm more closely would be useful.

Our study has at least three implications. First, the number of elements in the optic array may affect the speed and effectiveness of relative TTC judgments. Thus, results of two-object displays may not generalize to displays with more objects. Second, RT results suggest that processing load as well as optic expansion information must be included in models of perceived collision (DeLucia \& Blume, 1994); however, processing load may not be effective in detection tasks such as that in Experiment 2. Third, visual information other than optical TTC may contribute to relative TTC judgments; thus, it is important to determine the limits, relative strengths, and combinatorial rules for different sources of visual information.

It is important to point out several limits and caveats in the interpretation of our results. First, the conclusion 
that observers can judge which of as many as eight approaching objects would hit them first should be qualified. For example, although accuracy with eight-object displays was greater than chance and $d^{\prime}$ was greater with eight objects than with two, the results also suggested that information other than, and contingently related to, optical TTC contributed to performance. It is reasonable to propose that if performance is high when information other than optical TTC (e.g., global expansion) is contingently related to TTC, performance would be poor when such information is unrelated to, or contradicts, TTC. Thus, results may be more accurately characterized as suggesting that observers can judge which of as many as eight objects would hit them first when certain sources of visual information are consistent with TTC.

Second, although mean RT was smallest with twoobject displays (in Experiment 1), further study is needed to conclude that performance is constrained by limitedcapacity processing or that observers performed either a serial or parallel search of the objects. Specifically, the increase from two to eight objects was very small $(10 \mathrm{msec})$, and RT and $d^{\prime}$ results may reflect a speed-accuracy tradeoff. Further, RT was smallest with eight objects in the detection task of Experiment 2. Moreover, under certain conditions, it is possible for parallel and serial systems to mimic each other; for example, it is possible to build a serial model that mimics an independent parallel model with unlimited capacity (at some level; Townsend, 1974; Townsend \& Ashby, 1983). Our study was not designed to distinguish these models.

Finally, we note that our results are limited to the displays that we used (which involved many constraints) and that our experiments were not designed to examine individual differences or to determine the decision rules (see, e.g., Graham, 1989) that observers used. For example, observers may differ from one another in such decision rules and in the manner in which they use different sources of visual information. Nevertheless, our attempt to measure relative TTC judgments of as many as eight approaching objects produced systematic and informative results; further studies of this kind would be worthwhile.

\section{REFERENCES}

BaRLow, H. B. (1978). The efficiency of detecting changes of density in random dot patterns. Vision Research, 18, 637-650.

Bootsma, R. J., \& Oudejans, R. R. D. (1993). Visual information about time to collision between two objects. Journal of Experimental Psychology: Human Perception \& Performance, 19, 1041-1052.

BRADDICK, O. J. (1993). Adaptation and "pop-out" in the analysis of divergence-sensitive mechanisms. Perception, 22, 500.

BradDicK, O. J., \& HollidaY, I. E. (1991). Serial search for targets defined by divergence or deformation of optic flow. Perception, 20, 345-354

Cheal, M., \& Lyon, D. R. (1992). Attention in visual search: Multiple search classes. Perception \& Psychophysics, 52, 113-138.

CowAN, N. (1988). Evolving conceptions of memory storage, selective attention, and their mutual constraints within the human informationprocessing system. Psychological Bulletin, 104, 163-191.

DeLucia, P. R. (1991a). Pictorial and motion-based information for depth perception. Journal of Experimental Psychology: Human Perception \& Performance, 17, 738-748.
DeLucia, P. R. (1991b). Small near objects can appear farther than large far objects during object motion and self motion: Judgments of object-self and object-object collisions. In P. J. Beek, R. J. Bootsma, \& P. C. W. van Wieringen (Eds.), Studies in perception and action: Posters presented at the Sixth International Conference on Event Perception and Action (pp. 94-100). Amsterdam: Rodopi.

DELUCIA, P. R. (1995). Effects of pictorial relative size and ground-intercept information on judgments about potential collisions in perspective displays. Human Factors, 37, 528-538.

DeLuCia, P. R., \& Blume, J. L. (1994, May). Judging first arrival with two or eight approaching objects: Processing optic expansion information takes time. Poster presented at the annual meeting of the Association for Research in Vision and Ophthalmology, Sarasota, FL.

DeLuCiA, P. R., \& LIDDELL, G. W. (in press). Cognitive motion extrapolation and cognitive clocking in prediction-motion tasks. Journal of Experimental Psychology: Human Perception \& Performance.

DeLucia, P. R., \& WARren, R. (1994). Pictorial and motion-based information during active control of self-motion: Size-arrival effects on collision avoidance. Journal of Experimental Psychology: Human Perception \& Performance, 20, 783-798.

Duncan, J., \& Humphreys, G. W. (1989). Visual search and stimulus similarity. Psychological Review, 96, 433-458.

Elliot, P. B. (1964). Appendix 1-Tables of $d^{\prime}$. In J. A. Swets (Ed.), Signal detection and recognition by human observers (pp. 651-683). New York: Wiley.

EnNS, J., \& Rensink, R. A. (1990). Sensitivity to three-dimensional orientation in visual search. Psychological Science, 1, 323-326.

GraHaM, N. V. S. (1989). Visual pattern analyzers. New York: Oxford University Press.

Green, D. M., \& Swets, J. A. (1966). Signal detection theory and psychophysics. New York: Wiley.

HACKER, M. J., \& RATCLIFF, R. (1979). A revised table of d' for M-alternative forced choice. Perception \& Psychophysics, 26, 168-170.

Hochberg, J. E. (1978). Perception (2nd ed.). Englewood Cliffs, NJ: Prentice-Hall.

HOCHBERG, J. (1986). Representation of motion and space in video and cinematic displays. In K. R. Boff, L. Kaufman, \& J. P. Thomas (Eds.), Handbook of perception and human performance (pp. 22-1 to 22-64). New York: Wiley.

Hunt, E., Pellegrino, J. W., Frick, R. W., Farr, S. A., \& AlderTON, D. (1988). The ability to reason about movement in the visual field. Intelligence, 12, 77-100.

JAGACINSKI, R., Johnson, W., \& MilleR, R. (1983). Quantifying the cognitive trajectories of extrapolated movements. Journal of Experimental Psychology: Human Perception \& Performance, 9, 43-57.

Kahneman, D. (1973). Attention and effort. Englewood Cliffs, NJ: Prentice-Hall.

KAISER, M. K., \& MOWAFY, L. (1993). Optical specification of time-topassage: Observers' sensitivity to global tau. Journal of Experimental Psychology: Human Perception \& Performance, 19, 1028-1040.

KHURANA, B., \& KowLER, E. (1987). Shared attentional control of smooth eye movement and perception. Vision Research, 27, 1603-1618.

LARISH, J. F., \& ANDERSEN, G. J. (1995). Active control in interrupted dynamic spatial orientation: The detection of orientation change. Perception \& Psychophysics, 57, 533-545.

Law, D. J., Pellegrino, J. W., Mitchell, S. R., Fischer, S. C., McDonALD, T. P., \& HunT, E. B. (1993). Perceptual and cognitive factors governing performance in comparative arrival-time judgments. Journal of Experimental Psychology: Human Perception \& Performance, 19, 1-17.

LEE, D. N. (1974). Visual information during locomotion. In R. B. McLeod \& H. L. J. Pick (Eds.), Perception: Essays in honor of James J. Gibson (pp. 250-267). Ithaca, NY: Cornell University Press.

LuCE, R. D., Bush, R. R., \& GalANTer, E. (1963). Handbook of mathematical psychology (Vol. 1). New York: Wiley.

Macmillan, N. A., \& Creelman, C. D. (1991). Detection theory: A user's guide. Cambridge: Cambridge University Press.

MCLeOD, P., Driver, J., \& CrisP, J. (1988). Visual search for a conjunction of movement and form is parallel. Nature, 332, 154-155.

MCNicol, D. (1972). A primer of signal detection theory. London: Allen \& Unwin.

PaChella, R. G. (1974). The interpretation of reaction time in information- 
processing research. In B. H. Kantowitz (Ed.), Human information processing (pp. 41-82). Hillsdale, NJ: Erlbaum.

PALMER, J. (1990). Attentional limits on the perception and memory of visual information. Journal of Experimental Psychology: Human Perception \& Performance, 16, 332-350.

Pellegrino, J. W., \& Hunt, E. B. (1989). Computer-controlled assessment of static and dynamic spatial reasoning. In J. W. Pellegrino (Ed.), Testing: Theoretical and applied perspectives (pp. 174-198). New York: Praeger.

PollaCK, I. (1959). Message uncertainty and message reception. Journal of the Acoustical Society of America, 31, 1500-1508.

POLLACK, I. (1964). Message probability and message reception. Journal of the Acoustical Society of America, 36, 937-945.

PoSNER, M. I. (1980). Orienting of attention. Quarterly Journal of Experimental Psychology, 32, 3-25.

Previc, F. H., \& Blume, J. L. (1993). Visual search asymmetries in three-dimensional space. Vision Research, 33, 2697-2704.

ProctoR, R. W., \& VAN ZANDT, T. (1994). Human factors in simple and complex situations. Boston: Allyn \& Bacon.

REGAN, D. (1993). The divergence of velocity and visual processing. Perception, 22, 497-499.

Regan, D., \& Hamstra, S. J. (1993). Dissociation of discrimination thresholds for time to contact and for rate of angular expansion. $\mathrm{Vi}$ sion Research, 33, 447-462.

SCHIFF, W., \& DETWILER, M. L. (1979). Information used in judging impending collision. Perception, 8, 647-656.

SCHIFF, W., \& OLDAK, R. (1990). Accuracy of judging time to arrival: Effects of modality, trajectory, and gender. Journal of Experimental Psychology: Human Perception \& Performance, 16, 303-316.

ShIFFRIN, R. M. (1976). Capacity limits in information processing, attention, and memory. In W. K. Estes (Ed.), Handbook of learning and cognitive processes (Vol. 4, pp. 177-235). Hillsdale, NJ: Erlbaum.

SIMON, C. W. (1977). New research paradigm for applied experimental psychology: A system approach (Tech. Rep. TR CWS-04-77A). Westlake Village, CA: Canyon Research Group, Inc.

SImPSON, W. A. (1988). Depth discrimination from optic flow. Perception, 17, 497-512.

SPERLING, G., \& ReEves, A. (1980). Measuring the reaction time of a shift of visual attention. In R. S. Nickerson (Ed.), Attention and performance VIII (pp. 347-360). Hillsdale, NJ: Erlbaum.

TeICHNER, W. H., \& KREBS, M. J. (1974). Visual search for simple targets. Psychological Bulletin, 81, 15-28.

ToDD, J. T. (1981). Visual information about moving objects. Journal of Experimental Psychology: Human Perception \& Performance, 7, 795-810.

TOWNSEND, J. T. (1974). Issues and models concerning the processing of a finite number of inputs. In B. H. Kantowitz (Ed.), Human information processing: Tutorials in performance and cognition (pp. 133183). Hillsdale, NJ: Erlbaum.

TownSEND, J. T., \& AshBY, F. G. (1983). The stochastic modeling of elementary psychological processes. Cambridge: Cambridge University Press.

Treisman, A., \& Gelade, G. (1980). A feature-integration theory of attention. Cognitive Psychology, 12, 97-136.

Treisman, A., \& GoRmICAN, S. (1988). Feature analysis in early vision: Evidence from search asymmetries. Psychological Review, 95, 15-48.

Tresilian, J. R. (1990). Perceptual information for the timing of interceptive action. Perception, 19, 223-239.

Tresilian, J. R. (1991). Empirical and theoretical issues in the perception of time to contact. Journal of Experimental Psychology: Human Perception \& Performance, 17, 865-876.

Tresilian, J. R. (1995). Perceptual and cognitive processes in time-tocontact estimation: Analysis of prediction-motion and relative judgment tasks. Perception \& Psychophysics, 57, 231-245.

WANG, Y., \& Frost, B. J. (1992). Time to collision is signalled by neurons in the nucleus rotundus of pigeons. Nature, 356, 236-238

YaGer, D., Kramer, P., Shaw, M., \& Graham, N. (1984). Detection and identification of spatial frequency: Models and data. Vision Research, 24, 1021-1035.

\section{NOTES}

1. For brevity, the phrase time-to-contact will be used generically to denote the time remaining before an object contacts the observation point or a target, or passes it without collision.

2. Two prior studies measured judgments about which of four horizontally moving digits would arrive at a vertical line first, but did so to assess dynamic spatial reasoning (Hunt et al., 1988; Pellegrino \& Hunt, 1989).

3 . There are independent-channels models of detection and identification that predict a decrement in performance as a function of set size without assuming limited attentional capacity. Decrements are due to noise from the channels, which increases the rate of false alarms as set size increases (Graham, 1989; Yager, Kramer, Shaw, \& Graham, 1984). Our study was not designed to evaluate such models.

4. Regan and Hamstra (1993) reported higher discrimination thresholds than those obtained by Todd (1981), but their observers judged whether one object would arrive sooner than or later than the mean of a set of stimuli that was previously presented. We also note that Kaiser and Mowafy (1993) reported that judgments of relative time-to-passage (using simulations of self-motion) were reliable when differential timeto-passage in the displays was about $500 \mathrm{msec}$, whereas Todd (1981) demonstrated that the accuracy of relative TTC judgments (using simulations of object motion) was greater than $90 \%$ when differential TTC was as small as $150 \mathrm{msec}$. We selected a differential TTC of $200 \mathrm{msec}$ because our displays were more similar to Todd's (e.g., only global tau and not local tau was present in Kaiser and Mowafy's displays), and because relative TTC was specified veridically by local tau and global tau in our displays.

5. The boundary lines were provided to help observers identify and discriminate the objects' display locations. However, due to constraints in the scene design, the objects did not always move without contacting or extending partly beyond a boundary line, especially with more objects. Nevertheless, an object's assigned display area was clear.

6 . The latter cue is relevant because if two objects have the same velocity and are equidistant from the principal line of sight, the object that is closer to the observation point would leave the field of view first; when an object leaves the screen in a computer simulation, it can be interpreted as leaving the field of view.

7. Configuration was only partly crossed with FA location; for the horizontal configuration, the FA was located in areas 1,2,4, and 6; for the vertical configuration, the FA was located in areas $3,5,7$, and 8 . This was so for all set sizes. ANOVA results indicated that there were no significant interactions between configuration and any other factors.

8. Other results that appear to be inconsistent with Todd's (1981) have been reported previously (e.g., DeLucia, 1991a; Simpson, 1988; see also Law et al., 1993) and may be due to differences in display parameters.

9. We interpret the $d^{\prime}$ scores with certain caveats. First, such scores involve assumptions that the distributions of signal and noise are normal and of equal variance (Green \& Swets, 1966; McNicol, 1972). Second, measures of proportion correct and $d^{\prime}$ will be depressed in a forcedchoice method if observers use the response alternatives with different frequencies (Green \& Swets, 1966; Macmillan \& Creelman, 1991). In our study, although the FA was placed in each of the eight display areas for an equal number of trials, accuracy was higher when it was located in areas $4,6,7$, and 8 compared with the remaining scenes. Third, there was a relatively small number of trials in each condition (see Green \& Swets, 1966). Finally, we note that the target (signal) is defined as the FA, which is a relative parameter (smallest TTC).

10 . When proportion correct was 0 or 1.00 , we used .01 and .99 , respectively, in order to estimate $d^{\prime}$ (for alternative methods, see Macmillan \& Creelman, 1991). Also, for another example of analyzing $d^{\prime}$ with ANOVA, see Larish and Andersen (1995).

11. Probability values from the ANOVAs reflect Greenhouse-Geisser corrections. Factors that account for less than $1 \%$ of the variance are considered negligible and generally are not discussed (Simon, 1977; see also Schiff \& Detwiler, 1979).

12. The main effect of set size on mean RT for correct responses (averaged across FA location to avoid missing cells) was not significant. However, when results of two-object and eight-object displays were averaged 
across Experiment 1 and a control experiment (see note 14), mean RT for correct responses was greater with eight objects than with two [ $2.28 \mathrm{vs}$. $\left.2.42 \mathrm{sec} ; F(1,15)=8.37, M S_{\mathrm{e}}=.04, p<.0112, w^{2}=6.22 \%\right]$.

13. A trend analysis of the relationship between set size and performance indicated a significant linear component for $\mathrm{RT}[F(1,23)=9.88$, $\left.p<.005, R^{2}=4.11 \%\right]$ and $d^{\prime}\left[F(1,23)=121.93, p<.001, R^{2}=58.95 \%\right.$. $]$ The same results occurred when feedback was provided in a control experiment except that the quadratic component also was significant for RT.

14. A disadvantage of the method used in Experiment 1 is that speed and accuracy are not measured simultaneously. In two separate control experiments, observers viewed the two-object and eight-object displays in Experiment 1. In one case, 8 observers pressed one of eight keys on a computer keyboard (as quickly and as confidently as possible) to report the apparent location of the FA. In the second case, 8 different observers used the one-key, two-stage response as in Experiment 1. The effects of set size in both experiments were similar to those in Experiment 1; however, with the eight-key method, the effect of set size on $d^{\prime}$ occurred only in Set 2, and there was no effect of set size on efficiency. Results of an ANOVA indicated that effects of set size on RT, $d^{\prime}$, or efficiency did not differ between the experiments. However, a disadvantage of the eight-key method is that set size covaries with the number of relevant response selections, which potentially could inflate RT and errors with larger set sizes (for related issues, see Proctor \& van Zandt, 1994; Teichner \& Krebs, 1974). Thus, we used the one-key method in the remaining experiments. Finally, in a third control experiment, 8 new observers were given feedback regarding the specific location of the FA after every trial. Accuracy was significantly above chance in all conditions of set and set size $(p<.001)$; mean $d^{\prime}$ and RT were greater with eight or six objects than with two $(p<.05)$. Also, set size accounted for less than $1 \%$ of the variance, and there was no effect of set size on efficiency. Thus, observers may have used the feedback to overcome effects of relative size and to reduce set-size effects. However, feedback also may have led observers to use artificial response strategies that were not based on their perceptual experiences. Indeed, 2 observers reported that on at least some trials they made responses that were not consistent with their perceptual experiences in an attempt to enhance their accuracy on the basis of feedback. Thus, results cannot be interpreted with confidence.

15. For convenience, we discuss expansion rather than rate of expansion because the entire duration was the same in all of our scenes.

16. Barlow (1978) reported that statistical efficiency permits valid comparisons between performance at different tasks. Statistical efficiency is applicable only when a theoretical limit to performance can be calculated, and that limit should involve signal/noise ratios (Barlow, 1978). It is reasonable to assume that our task fulfilled such constraints because observers searched for one object among a variable number of objects and we used $d^{\prime}$ as a measure of performance.

17. An alternative explanation of the set-size effects on RT is that observers successively fixate each object; greater set sizes require more fixations, thereby inflating RT. Eye movements were not measured and are difficult to control experimentally. Within the visual-search paradigm, it has been reported that an increase in RT as a function of set size can occur even when eye movements are ruled out with short displays (Treisman \& Gormican, 1988). It was not practical to use brief displays with our moving scenes. Moreover, an attempt to eliminate the role of eye movements would not prevent attentional shifts in the absence of fixations (see, e.g., Posner, 1980; Sperling \& Reeves, 1980), which could also lead to longer RTs with larger set sizes.

(Manuscript received March 7, 1996; revision accepted for publication August 28, 1996.) 